\title{
La correspondencia entre Ricardo Molina y José Manuel Caballero Bonald con la revista Cántico y el flamenco de fondo*
}

\section{Exploring the Correspondence between Ricardo Molina and José Manuel Caballero Bonald through the Journal Cántico and Flamenco Music}

\author{
José Jurado Morales \\ Universidad de Cádiz \\ jose.jurado@uca.es \\ ORCID iD: https://orcid.org/0000-0002-6633-6362
}

\section{RESUMEN}

En este artículo presentamos y analizamos la correspondencia inédita entre los escritores Ricardo Molina (1917-1968), miembro del grupo Cántico de Córdoba, y José Manuel Caballero Bonald (1926), premio Cervantes 2012. En primer lugar, hacemos referencia a la amistad entre ambos generada en torno a la revista Cántico. Comentamos una carta de 1955 en la que Molina invita a Caballero Bonald a participar en el famoso homenaje de Cántico a Luis Cernuda. En segundo lugar y más extensamente, profundizamos en las relaciones que mantienen motivadas por la atracción compartida por el flamenco. Referimos su labor como flamencólogos y comentamos varias cartas inéditas de 1963-1965 en las que ellos hablan de la Semana de Estudios Flamencos de Málaga, el Concurso de Flamenco de Córdoba, Antonio Mairena y Mauricio Ohana, entre otros aspectos. Ciertamente el análisis de estas cartas ayuda a ahondar en el perfil de Ricardo Molina y José Manuel Caballero Bonald como flamencólogos.

Palabras Clave: Ricardo Molina; José Manuel Caballero Bonald; Pablo García Baena; Cántico; flamenco; Luis Cernuda.

\section{ABSTRACT}

In this article we shall present and analyse the unedited correspondence between the writers Ricardo Molina (1917-1968), member of the group Cántico de Córdoba, and José Manuel

* Este artículo se halla vinculado al Proyecto de Investigación del Plan Estatal «Poéticas de la Transición (1973-1982)» financiado por FEDER/Ministerio de Ciencia, Innovación y Universidades - Agencia Estatal de Investigación/ FFI2017-84759-P. 
Caballero Bonald (1926), Premio Cervantes 2012. First of all, we shall discuss the friendship that surged between these two writers as a result of their relationship with the review, Cántico. More specifically, we shall reference the letter Molina sends Caballero Bonald in 1955 in which he invites Caballero Bonald to participate in Cántico's famous homage to Luis Cernuda. Secondly, and more extensively, we shall elucidate upon the relationship they maintained due to their shared interest in flamenco music. We shall reference their work as flamencologists and further comment upon the collection of letters 1963-1965 in which they discuss, amongst other topics, the Week of Flamenco Studies, Málaga, the Córdoba Flamenco Contest, Antonio Mairena and Mauricio Ohana. Certainly, the analysis of this unedited collection of letters shall help to understand more the profiles of both Ricardo Molina and José Manuel Caballero Bonald as experts in flamenco.

Key words: Ricardo Molina; José Manuel Caballero Bonald; Pablo García Baena; Cántico; Flamenco; Luis Cernuda.

Ricardo Molina y José Manuel Caballero Bonald coinciden por primera vez en Córdoba a finales de 1950. Desde ese primer encuentro se mantiene la relación amistosa entre ambos como se aprecia en varios detalles biográficos y librescos. Así, por ejemplo, en la biblioteca de Caballero Bonald se halla un ejemplar de Elegías de Sandua, la separata del primer número extraordinario de la revista Cántico de enero de 1948, a la que considera «la cima para mí de su obra poética y cuya edición de 1948 conservo como oro en paño» (Caballero Bonald 2008, 100). Por su lado, entre los volúmenes que se conservan de la biblioteca particular de Molina se encuentran ejemplares de las primeras ediciones de dos poemarios iniciales de Caballero Bonald: Memorias de poco tiempo y Anteo. El primero, estampado en Ediciones Cultura Hispánica en 1954, lleva esta dedicatoria: «A Ricardo Molina, con la devoción y el seguro abrazo de JoséManuel / Madrid, 1.VI.54». Por su lado, Anteo se publica en la colección de Los Papeles de Son Armadans en 1956 con una tirada de cien ejemplares «destinados a los amigos del autor», según se especifica en las páginas interiores. El depositado en la biblioteca de Molina lleva manuscrito el número 46 de la tirada y una dedicatoria que reza: «A Ricardo Molina, que conoce mejor que yo este planeta, con mi leal abrazo de siempre, / JoséManuel / Palma de Mallorca, 20.IX.56».

Ese «leal abrazo de siempre» se alarga hasta principios del siglo XXI como atestigua la participación prevista del escritor jerezano en las jornadas literarias organizadas por el Ayuntamiento de Córdoba en marzo de 2007 para conmemorar el noventa aniversario de su nacimiento. Digo prevista porque, según Julio Neira (2014, 524), Caballero Bonald no acude a pronunciar la conferencia de clausura el día 8 porque se lo impide una gripe. En todo caso, el texto se publica en las actas del congreso con el título de «Ricardo Molina y el flamenco» y ahí leemos en su comienzo: 
Me agrada de manera muy especial venir a Córdoba a hablar de Ricardo Molina y, en particular, de su notable labor como experto en temas flamencos. Agradezco a los organizadores que hayan pensado en mí, y no solo por el gusto de referirme a unas cuestiones cuyo interés compartí con Ricardo, sino porque se trata de un poeta al que continúa uniéndome una devoción intacta (Caballero Bonald 2008, 99).

Igualmente la correspondencia conservada entre ambos da cuenta de unos lazos que perduran hasta la muerte del poeta cordobés. En el archivo de la Fundación Caballero Bonald se guardan seis cartas de Ricardo Molina: una datada en 1955, cuatro fechadas entre setiembre y octubre de 1963 y una última de agosto de 1964. En el archivo de Ricardo Molina solo he encontrado una de Caballero Bonald correspondiente a abril de 1965. Estos datos cronológicos arrojan una conclusión de entrada: hay una carta descolgada en 1955, hay varias concentradas en 1963 y hay otras dos desperdigadas de 1964 y 1965. Y apuntan otra conclusión: no podemos establecer el diálogo entre los corresponsales al faltar las contestaciones de Caballero Bonald. Con todo, este corpus epistolar permite ahondar en las relaciones vitales, culturales y literarias entre uno y otro del mismo modo que ayuda a perfilar y profundizar en la biografía y los intereses intelectuales de Ricardo Molina. En las páginas que siguen daré a conocer y comentaré esta correspondencia inédita. En primer lugar, haré mención a la etapa de Cántico y a la relación de ambos como poetas, y, en segundo lugar, me detendré en el mundo del flamenco, por el que los dos sintieron una atracción artística e intelectual común.

\section{Las relaciones amistosas entre Ricardo Molina y Caballero Bonald a TRAVÉS DEL GRUPO Y LA REVISTA CÁNTICO}

La prehistoria de la relación entre los integrantes del grupo Cántico y José Manuel Caballero Bonald se remonta a la primavera de 1947 cuando Pablo García Baena se tropieza con el jerezano Juan Valencia en Córdoba (García Baena 2007; Neira 2014, 94-96). Aquel encuentro abre las puertas de la revista Cántico a los jóvenes gaditanos que por entonces comienzan a escribir. Al inicio de la conferencia mencionada «Ricardo Molina y el flamenco» Caballero Bonald expresa que «Empezaré por recordar que el primer tramo de mi biografía como poeta está unido a Cántico, a Pablo García Baena, a Juan Bernier, a Julio Aumente y, por supuesto, a Ricardo Molina» (Caballero Bonald 2008, 99).

La correspondencia entre García Baena y Caballero Bonald testimonia el interés mutuo porque el jerezano publique en Cántico e informa del envío de algunos de sus poemas en 1948 así como de la recepción entusiasta y del propósito de publicarlos por parte de aquel en 1949. Sin embargo, nunca llegan a ver la luz. El 6 de diciembre de 1948 García Baena le escribe lo siguiente: 
Gracias, muchas gracias por los maravillosos poemas que has mandado para «Cántico». [...] Los poemas me han gustado extraordinariamente y mi primer impulso fué que salieran inmediatamente en nuestro número de Diciembre; pero en dicho número por estar ya casi completo no podríamos darte más que un solo poema. He preferido que salgan en Febrero y te dedicaremos una página completa.

Me encanta que seamos nosotros los primeros en dar tus versos (supongo que hasta ahora apenas habrás publicado) pues nuestro mayor anhelo es dar a conocer jóvenes valores de la poesía y preferimos siempre a los andaluces. Cuando los poemas son tan buenos como los tuyos nuestra alegría es inmensa.

El 17 de marzo de 1949 vuelve García Baena sobre la cuestión de publicar a Caballero Bonald en los siguientes términos: «Supongo recibirías el $n^{\circ} 8$ de "Cántico" y el extraordinario de Juan Bernier. Y estarías esperando el no 9 con tus poemas como te prometí. Pero ahora damos otro extraordinario de Gabriel Celaya y así el número normal donde van dos de tus poemas no saldrá hasta Mayo».

Unos meses después, el 21 de setiembre de 1949, García Baena dice responderle a una carta de Caballero Bonald de mayo y le subraya que

«Cántico» atraviesa por un largo marasmo, del que no sabemos si saldrá. Hemos tenido un tropiezo con la censura. La revista salía sólo con una autorización particular, que ha sido anulada por otra orden más alta. Te ruego no cuentes esto más que a tus íntimos. Tenemos esperanzas de que no pase el año sin lanzar otro número que se nos quedó a medio componer y en el cual, por fin, irán tus poemas.

Estos fragmentos de la correspondencia de García Baena a Caballero Bonald indican que este se queda sin publicar en la etapa inicial de Cántico, es decir, entre el número uno, de octubre de 1947, y el ocho, de diciembre de 1948 y enero de 1949.

Al margen de estas cartas cruzadas entre 1948 y 1949, según cuenta Caballero Bonald en sus memorias, el primer encuentro físico entre ellos tiene lugar a finales de 1950 durante una visita del grupo gaditano (Fernando Quiñones, Pilar Paz Pasamar y el propio Caballero Bonald, entre otros) para realizar un encuentro poético con los cordobeses. De aquella visita, de lo que hacen y de la amistad que comienzan a trabar los de Cántico y Caballero Bonald, ha dejado constancia Pablo García Baena en una carta sin fechar en la que acusa recibo de una tarjeta de Caballero Bonald en la que este refiere lo vivido y disfrutado en Córdoba. Muchos años después, en sus memorias de 1995, el jerezano reconstruye la impronta que los cordobeses le dejan:

Yo no conocía a los poetas de Cántico -aunque sí había leído algo de ellos- y creo que ésa fue la primera vez que me encontré, fuera de mi órbita nativa, con unas personas que se identificaban plenamente con mi bagaje de inocencias a propósito del comportamiento de un creador. Me refiero en especial a Pablo García Baena. Ricardo Molina era algo espeso de trato, un poco socarrón y monocorde. Julio Aumente y Miguel del Moral parecían, cada uno a su aire, miembros 
de una sociedad secreta juramentados para soliviantar a bienpensantes y Juan Bernier no hacía más que divagar por unos circunloquios mentales ligeramente perversos (Caballero Bonald 2004, 170-171).

Los efectos literarios de aquel bien avenido encuentro de finales de 1950 muy pronto se hacen notar, pues el número 1 de la segunda etapa de la revista gaditana Platero, fechado en enero de 1951, se abre con el poema «Oda a Luis Cernuda» de Ricardo Molina, que vuelve a colaborar con «Tristán» en el número seis, de junio de 1951, y con «Primeras lluvias» en el número catorce, de febrero de $1952^{1}$. Refiero lo de Platero porque evidencia el compañerismo en las aventuras poéticas compartidas y porque supone un eslabón adelantado del interés de Molina y de sus colegas cordobeses por restituir la persona y la obra de Cernuda en la España franquista. Y por algo más: porque el poeta sevillano engendra la razón de la primera de las cartas conservadas de Ricardo Molina enviadas a Caballero Bonald. Es breve en la extensión y muy concreta en el contenido, pero se antoja muy significativa porque alude al momento cenital de la segunda época de Cántico en la historia de la poesía española de posguerra: el número extraordinario de homenaje a Luis Cernuda. Corresponde a 1955, aunque no se precisa la fecha exacta, y Ricardo Molina le dice:
Querido amigo: Te requiero con el máximo empeño para que colabores con unos tres ó cuatro folios en prosa a doble espacio en el Homenaje Andaluz a Luis Cernuda que entrará en prensa el 15 de Noviembre. Es el número que le dedica Cántico. Tú no puedes faltar. Te agradecería que me escribas dándome seguridad de tu colaboración.
Un fuerte abrazo
Ricardo Molina
T/C. Cascajo 26

Ha sido investigado por extenso el valor trascendente que supone el número 9-10 de Cántico, correspondiente a agosto-noviembre de 1955, para la poesía española y para la reincorporación de Cernuda al panorama cultural de la posguerra. Por lo escueto del mensaje y lo preciso de las indicaciones, parece apuntar a un modelo de carta que Molina redacta y envía a todos los que invita a colaborar. Asimismo, la carta manifiesta la responsabilidad constante de Molina al frente de la revista, tal y como han atestiguado algunos de sus estudiosos más fervientes (Carnero 1976, 2008; Torre 1995; Rendón Infante 2015). No he encontrado contestación alguna de Caballero Bonald en el archivo del cordobés y, en última instancia, lo que queda claro al consultar el número de la revista es que este no colabora en el homenaje a Cernuda ${ }^{2}$, como tampoco

${ }^{1}$ Ricardo Molina 1951a; 1951b; 1952; 1982, 111-113; 2007, 203-205.

2 El homenaje de Cántico cuenta con textos de Federico García Lorca, Vicente Aleixandre, Manuel Altolaguirre, José María Pemán, Adriano del Valle, Julio Aumente, Muñoz 
lo hace finalmente en toda la historia de Cántico, ni en la primera época entre octubre de 1947 y diciembre de 1948 y enero de 1949 ni en la segunda entre abril de 1954 y 1957.

\section{Ricardo Molina y Caballero Bonald con el flamenco De Fondo}

José María de la Torre sostiene en su libro Ricardo Molina. Perfil de un poeta que el agotamiento de Cántico ya se percibe desde el número de homenaje a Luis Cernuda y arguye como motivos principales el posible cansancio de Molina y su descubrimiento de horizontes culturales nuevos, entre ellos el del flamenco, algo que también influiría en el descenso de su producción poética a partir de la publicación de Corimbo en 1949 (Torre 1995, 77-98). Sean estas las razones últimas u otras, tal y como han indicado algunos críticos (caso de Abelardo Linares 1983 y Luis Jiménez Martos 1990), cito la reflexión de José María de la Torre $(1995,54)$ porque justamente el flamenco constituye el nexo de unión en la correspondencia entre Molina y Caballero Bonald:

Las causas de su desaparición [de Cántico], ahora sí definitiva, hay que imputárselas al propio R. Molina, porque, aparte de su posible cansancio por mantener el cometido con su solo esfuerzo personal, descubre horizontes culturales nuevos, entre los que se encuentra el mundo telúrico y ancestral del Flamenco, según prueban los artículos sobre el tema publicados en noviembre de 1955.

Tanto Ricardo Molina como José Manuel Caballero Bonald han pasado a engrosar la lista de los flamencólogos más estimados y citados que se dan a conocer a partir de mediados del siglo XX, como Anselmo González Climent ${ }^{3}$, José Monleón, Ángel Álvarez Caballero, Fernando Quiñones, Manuel Ríos Ruiz, Félix Grande, Agustín Gómez Pérez, José Blas Vega, José Manuel Flores y José Luis Ortiz Nuevo, entre otros. Se ha escrito mucho del conocimiento y del interés de Ricardo Molina por el flamenco que dan de sí como resultados mayores Mundo y formas del cante flamenco (1963b), escrito en colaboración con Antonio Mairena y con el que obtiene el premio de investigación de la Cátedra de Flamencología de Jerez de la Frontera en 1964, Cante flamenco (1965) y Misterios del arte flamenco. Ensayo de una interpretación antropológica (1967), proyecto becado en la II Semana de Estudios Flamencos de Má-

Rojas, Fernando Quiñones, José Luis Cano, Ricardo Gullón, Enrique Azcoaga, Manuel Álvarez Ortega, Vicente Núñez, Leopoldo de Luis, Juan Bernier, García Baena, Mario López, Salvador Moreno, Ricardo Molina y Luis Cernuda.

${ }^{3}$ Para muchos de los aspectos mencionados aquí, como por ejemplo la Semana de Estudios Flamencos de Málaga así como el Concurso Nacional de Cante en Córdoba, y dada la proximidad entre las fechas tratadas y el año de su publicación, véase la Bibliografía Flamenca de Anselmo González Climent (1965). 
laga y último libro suyo publicado en vida; a los que hay que sumar las recopilaciones póstumas de artículos Obra flamenca (1977b) y Cante y Cantaores cordobeses (1977a).

Y también se sabe del interés y del conocimiento de Caballero Bonald por el flamenco manifiestos de forma particular en el opúsculo poético Anteo (1956), las breves notas de El cante andaluz (1953), el libro divulgativo El baile andaluz (1957), el ensayo Luces y sombras del flamenco (1975) y en la tan aplaudida recolección de grabaciones dispuestas en el Archivo del Cante Flamenco (1968), premio en la categoría de disco de la Cátedra de Flamencología de Jerez de la Frontera en 1969, refundido y aumentado en Medio siglo de cante flamenco, Premio Nacional del Ministerio de Cultura 1987. A modo de tributo por todas estas aportaciones salió a finales de 2016 el disco-libro Jerez a Caballero Bonald. Homenaje Flamenco y Literario de la Ciudad al Poeta que recoge la interpretación de una selección de textos y poemas de Caballero Bonald por parte de artistas locales de reconocido prestigio y la edición de varios artículos y poemas de escritores y periodistas en torno al autor ${ }^{4}$.

El caso es que los años centrales de la producción ensayística de Molina sobre el flamenco (1963-1967) y los años de los viajes y grabaciones de Caballero Bonald por los rincones de Andalucía (1963-1965) coinciden con las fechas de las cartas cruzadas entre uno y otro, por lo que no extraña que el asunto medular se ciña a cuestiones ligadas al flamenco.

\section{La I Semana de Estudios Flamencos de Málaga, Antonio Mairena y Mauricio OHana}

La I Semana de Estudios Flamencos de Málaga, que comienza el lunes 21 de octubre de 1963, genera las cuatro cartas de Molina escritas en un intervalo de algo menos de un mes: entre el 15 de setiembre y el 12 de octubre. Con el referente del Concurso del Cante Jondo de 1922 en Granada, el Ayuntamiento de Málaga con la colaboración de la Peña Juan Breva patrocina un encuentro de flamencólogos y flamencos en la Casa de la Cultura (Acquaroni 1963). Caballero Bonald, que había pronunciado unos meses antes, el 31 de mayo de 1963, una conferencia sobre los «Cantes grandes de Jerez» en Málaga, se in-

${ }^{4}$ El proyecto cuenta con la dirección artística y musical de José María VelázquezGaztelu y David Lagos. Participan con su voz y su música Paco Cepero, Manuel Moneo, Vicente Soto, José Mercé, María José Santiago, Tomasa Guerrero «La Macanita», Alfredo Lagos, David Lagos, Ismael Jordi, Rosario Montoya «la Reina Gitana», Jesús Méndez y Manuel Valencia. Colaboran con sus poemas y textos Velázquez-Gaztelu, Felipe Benítez Reyes, Pilar Paz Pasamar, Enrique Montiel, Jesús Fernández Palacios, José Ramón Ripoll, Luis García Montero, Joaquín Sabina, José María García López, Josefa Parra y Juan José Téllez. 
volucra en los actos de esta I Semana ${ }^{5}$ y en una carta que no he localizado debe invitar a Molina a que participe con una conferencia. Esto abre el diálogo entre ellos para ajustar los términos de su participación y para que Molina medie en la configuración de los actos.

En realidad, Molina no habla del contenido de su charla y se centra en aspectos relacionados con las circunstancias del viaje y de la pernoctación, tal y como se atisba en los siguientes pasajes que selecciono. Comienza la primera de sus cartas, del 15 de setiembre de 1963, confirmando su participación: «Te repito mi agradecimiento por haberte acordado de mí para los Estudios Flamencos y te ratifico mi conformidad». Y un poco más abajo le hace saber: «Yo pienso ir a Málaga la víspera, el 23, y volver a Córdoba en algún tren del 25 por la tarde. Supongo que los gastos de hotel que ofrecen los organizadores se extenderán a las fechas que, en principio, estimo necesarias de mi estancia en Málaga». En la carta del 21 de setiembre leemos: «No quiero perderme la fiesta de Marbella. En tus manos encomiendo mi deseo». Y en la cuarta, del 12 de octubre, le especifica: «Deseo que si nos mandáis [se refiere a Antonio Mairena] al mismo Hotel, creo que Miramar, me reserven habitación individual porque soy lector nocturno y "camero" extraordinariamente molesto para cualquier durmiente normal. / Yo partiré el 23 por la tarde y deseo estar en Málaga hasta el domingo 27, en que regresaré a Córdoba». Asimismo, Molina comienza esta carta del 12 de octubre de 1963 mencionando que le ha enviado un cuestionario para algo relacionado con su intervención en la I Semana de Estudios Flamencos de Málaga: «Te adjunto las respuestas al cuestionario con foto que te ruego encarecidamente rescates una vez hecho el fotograbado pues tengo vivo interés en conservarla. Como he respondido desbocadamente, podas lo que encuentres largo o sin interés»).

Esto es todo lo que Ricardo Molina menciona de su participación en el primer encuentro de Málaga, de manera que por estas cartas no logramos saber cuáles conformarán las directrices y temas de su charla. Ahora bien, gracias a las crónicas telefónicas del escritor y periodista madrileño Antonio Díaz-Cañabate aparecidas en la edición de Andalucía de $A B C$ tenemos conocimiento de cómo se desarrolla esa Semana de Estudios Flamencos y, en particular, del acto en el que interviene Ricardo Molina. Díaz-Cañabate (1963) hace un completo resumen de la charla de Molina en su crónica titulada «Las "siguiriyas" de Manuel Torres» del sábado 26 de octubre de 1963. Escribe así:

Estoy en el salón de la Casa de Cultura de Málaga, que rebosa de gente. Las ocho y media de la noche. Empieza la cuarta lección de la Semana de Estudios Flamencos. Habla Ricardo Molina, poeta y escritor cordobés. Buen poeta, del que

${ }^{5}$ Caballero Bonald interviene el 25 de octubre sobre «Ríos y afluentes del baile flamenco» con el acompañamiento de Micaela Flores Amaya, La Chunga, que baila un taranto y una rumba. 
conozco sus «Elegías de Sandua». Ricardo Molina comienza a hablar con llaneza. No es un conferenciante. No es un orador. Es un contertulio de una peña muy numerosa que perora acerca de la formación de los cantes primitivos. Don José Ortega y Gasset decía que los españoles han aprendido todo lo que saben en las tertulias. En esta gra[t]ísima tertulia malagueña, esta noche he aprendido de flamenco más que en toda mi vida. Ricardo Molina habla con poco empaque y mucha enjundia. De muchas tertulias he formado parte. En ninguna me encontré a nadie que hablara con tanta seguridad, conocimiento y galanura como Ricardo Molina. No tengo el honor de conocerle personalmente, pero, desde aquí, le envío mi gratitud. Gracias, Ricardo Molina. Por usted he sabido que las palmas, ese acompañamiento tan saleroso para ciertos cantes, ya las conocían los antiguos egipcios. Que según don Manuel de Falla hay una analogía entre los cantes hindúes y el flamenco. Que tal vez exista en él una influencia judía. Que desde el siglo XIII al XV persiste en España el influjo de la música arábigo-andaluza. Que en el XV entran en la península los gitanos, por el Pirineo. Que el pueblo que, como el gitano, forja los metales, es mago y dominan misteriosamente el cante y el baile. Que los gitanos que arribaron a Andalucía se compenetraron con su ambiente, quizá por la persistencia en esta tierra de los moriscos. Que del XVI al XVIII no se habla de flamenco. Es en tiempos de Carlos III cuando se inicia. Que en ninguna parte del mundo los gitanos cantan flamenco. Sólo en España. Que los cantes flamencos primitivos son la 'toná', la 'siguiriya' y los romances. Que el primer cantaor del que se tiene noticia se llamó Luis el de la Juliana. Luego el 'Planeta', maestro del 'fillo'. Y Ricardo Molina hace una pausa y nos anuncia que Antonio Mairena nos va a cantar una 'toná' sin guitarra, porque la aparición de la guitarra como acompañamiento del cante es muy posterior.

La detallada información y la valoración aportada por Díaz-Cañabate explican muy bien que a la altura de 1963, el año de publicación de Mundo y formas del cante flamenco, Ricardo Molina ha alcanzado un crédito sobresaliente como flamencólogo. Otra cosa es que con el paso del tiempo sus estudios y sus teorías se tilden de parciales o tendenciosas por su filiación mairenista (Gómez Pérez 2008; Molina 1992).

Como he apuntado, en la correspondencia enviada a Caballero Bonald no alude a los términos de su conferencia, en cambio se ocupa por extenso de mediar para que Antonio Mairena y Mauricio Ohana participen en la semana malagueña, algo que sí hará el primero y no el segundo.

Tanto en el archivo de Ricardo Molina como en el de José Manuel Caballero Bonald se guardan muchas cartas de Antonio Mairena. Se ha escrito bastante sobre la amistad entre Mairena y Molina al igual que de cómo las ideas y las teorías de Molina acerca del flamenco están influidas por el pensamiento y la personalidad de Mairena. No voy a entrar en esto, pues no toca aquí, y voy a limitarme ahora a recoger los pasajes de las cartas de Molina a Caballero Bonald que alumbran el papel jugado por el cordobés para que Mairena cantase en esa I Semana de Estudios Flamencos. Ya en la primera conservada, en la del 15 de setiembre, tras aceptar la invitación de Caballero Bonald, le anota: 
Dos letras para sugerir que si habéis pensado complementar conferencia con cantes, tanto a Mairena como a mí nos agradaría formar pareja para coincidir en Málaga y además porque solo Mairena podría interpretar con decoro y pureza los diez o doce cantes primitivos que aún conocemos.

En la segunda, la del 21 de setiembre, solo encontramos un añadido (en tinta negra diferente al tono verde del cuerpo de la carta) en el extremo superior izquierdo de la primera página donde destaca: «Esta tarde veré en Córdoba a Mairena y trataré el asunto». Esta escueta indicación hace sospechar con fundamento de la existencia de una carta no localizada en la que Caballero Bonald accede a que Mairena acompañe la conferencia de Molina. Solo dos días después, el 23 de setiembre, Molina le escribe una carta de una sola página en la que, salvo tres líneas finales en la posdata, trata exclusivamente de este asunto. Se expresa así:

El domingo 22 hablé con Mairena. Pide 25.000 ptas. por actuar el 24 y 26, más que le paguen hotel los días 23 al 26 inclusive. Te escribo a prisa para dar tiempo a 'los tratos' que puedan surgir entre Mairena y organizadores. Debes escribirle tú y acordar con él directamente las condiciones, recalcando que este ciclo aspira a ser anual.

La cuarta carta, del 12 de octubre, ofrece indicios para pensar que a Caballero Bonald debe parecerle alta la cantidad pedida por Mairena, pues Molina le refiere que «Hablé por teléfono con Mairena que está dispuesto a rebajar a 20.000 ptas.» y luego le menciona su deseo, que ya he comentado más arriba, de no compartir habitación con él.

Finalmente, Antonio Mairena interviene el mismo día que Molina y su actuación debe resultar excelente, según se deduce del encomio de Díaz-Cañabate (1963) en su crónica de $A B C$. Este recuerda el impacto, «la "jonda" emoción», que le causó escuchar, en compañía de Ignacio Sánchez Mejías en el cuarto de un colmao de Sevilla, a Manuel Torres cantando unas siguiriyas y que desde entonces nadie (ni Antonio Chacón ni Pastora Pavón ni ningún otro) le ha emocionado tanto. Por esta confesión, podemos valorar en mucho el hecho de que la actuación de Mairena en Málaga le rememorase aquella otra de Manuel Torres. Retomo la cita de la crónica de Díaz-Cañabate (1963, 64) en la que Molina da paso a Mairena:

Y Ricardo Molina hace una pausa y nos anuncia que Antonio Mairena nos va a cantar una 'toná' sin guitarra, porque la aparición de la guitarra como acompañamiento del cante es muy posterior. Antonio Mairena no parece un cantaor. Su calva, su aspecto es el de un buen burgués, jefe de Negociado. Pero, amigos, ¡cómo canta mi insigne tocayo! ¡Con qué pureza, con qué expresión, con qué refinado gusto! Nos embelesa. Nos conmueve. ¡Prodigio del flamenco puro! Ricardo Molina dice que conoce más de cincuenta 'siguiriyas' diferentes. Y uno se queda asombrado. ¡Cualquiera aprende flamenco así como así! Antonio Mairena 
va a cantar la sola 'siguiriya' que se conoce de 'el Planeta', y luego unas viejas trianeras. Me recordó a Manuel Torres, no les digo a ustedes más. El otro cante fundamental es la 'soleá', muy tardía, que no surge hasta mediados del XIX, siendo 'la Andonda', gitana de Triana, la mejor cantaora de esa época. Y oímos a Antonio Mairena una 'soleá'. Y este cante se nos ahonda, nos llega a lo hondo, a la hondura del sentimiento, y nos la llevamos en el alma por las calles de Málaga, donde ya impera la soledad de la noche, tan maravillosa como el cante flamenco.

Por otro lado, las cartas de 1963 revelan el interés de Molina por que participe Mauricio Ohana, pianista y compositor francés de origen sefardí y padre gibraltareño, nacido en Casablanca en 1913 y fallecido en París en 1992. El cordobés insiste, tanto en la correspondencia con Caballero Bonald como en otras, en que Ohana es el músico que más sabe de flamenco. En efecto, Ohana investigó sobre diversas músicas, entre ellas las músicas árabes y especialmente el flamenco. No ha de extrañar esta insistencia pues Mairena y Molina se sirven del asesoramiento técnico de Ohana, además de los de Narciso Yepes y Odón Alonso, para la realización de Mundo y formas del cante flamenco. Asimismo, Ohana forma parte del jurado del III Concurso Nacional de Cante en Córdoba en 1962 y del IV Concurso en 1965 (Gómez Pérez 2006). Por consiguiente, la relación entre Molina y Ohana era fluida.

En la primera de estas cuatro cartas, del 19 de setiembre, Molina le adelanta a Caballero Bonald:

Sin pecar de entrometido creo que puedo sugerirte por si os interesa la conveniencia de invitar a algún músico capacitado para hablar 'en músico' del cante: Hay uno, excepcional, el único que está enterado -e interesado- en el cante: Es Mauricio Ohana, genuino sucesor de Falla, genial compositor. Su dirección es

Ohana Mauricio

31, rue du General Delestraint

C/O. Madame Bot

PARIS (XVI)

Ohana es andaluz. Supongo conocerás su música para un entremés de Cela, su Prometeo, su «Llanto por Ignacio Sánchez Mejías» sobre el poema de Lorca, para Orquesta Coros y Recitador, etc...

El fue mi asesor musical para el libro sobre cante. Sería un nombre prestigioso en el programa de conferenciantes. [...] En caso de invitar a Ohana agradeceré que actúe el 23 o 25 para irnos juntos y poder oírlo.

La siguiente, la del 21 de setiembre, está centrada en Mauricio Ohana, salvo un párrafo final de seis líneas. Dice así:

Ohana, al que debes escribir a París, probablemente pasará unos días en Madrid bien este mes o el que viene. A lo mejor se pueden coordinar fechas -Ohana el 25, yo el 24. Caso de estar en Madrid la cosa se abarataría mucho. De todas formas conviene le escribas para que pueda preparar su conferencia que pudiera 
ser, dada la precipitación «El cante flamenco considerado o enjuiciado por un músico». Algo parecido, si lo deseas.

Ohana iría a Málaga en similares condiciones que yo, incluso debiendo viajar desde París, cuyo viaje en avión deberían pagar los organizadores. Creo que vale la pena. Prestigiaría el curso. ¿Habéis pensado en que hable algún pintor? Pienso en Moreno Galván, por ejemplo.

En la escueta carta de solo dos días más tarde, del 23 de setiembre, dedicada a la mediación con Mairena, solo menciona este asunto en la posdata: «Narciso Yepes te podría acaso informar en qué fechas estará Ohana en Madrid». Y en la siguiente y última conservada antes de la celebración de la Semana de Estudios Flamencos ya no le nombra a Ohana. Intuyo que la gestión para que acuda debe de complicarse puesto que no comparece en el programa definitivo $^{6}$. Sí aparece Ohana en el programa de la III Semana con una conferencia titulada «Incidencias del Arte Flamenco en la Música Actual», impartida el jueves 9 de setiembre de $1965^{7}$.

\section{La II Semana de Estudios Flamencos de Málaga, la Beca de Investigación y EL Concurso de Córdoba}

Como hemos visto, la invitación inicial de Caballero Bonald para que Molina dé una conferencia en la I Semana de Estudios Flamencos de Málaga logra sus frutos de modo bastante inmediato en ese mismo año de 1963. También participa e incluso con mayor implicación en la II Semana correspondiente a 1964 (Cifra 1964; Tijeras 1964; «Homenaje a Pastora Imperio en la II Semana de Estudios Flamencos» 1964; «Homenaje a Pastora Imperio» 1964), porque dirige un seminario y porque resulta premiado con una ayuda para realizar un estudio ligado al cante, el toque y el baile flamencos.

${ }^{6}$ José Blas Vega y Manuel Ríos Ruiz (1988) apuntan en el Diccionario enciclopédico ilustrado del flamenco el programa de la I Semana: Día 21, inauguración por el alcalde Francisco Bejarano; y coloquio sobre el tema dirigido por José Mercado Ureña. Día 22, «Significación popular del cante jondo», conferencia por José y Jesús de las Cuevas; actuación de los finalistas del Concurso de Cantes de Málaga, presentados por Enrique Crooke Caffarena. Día 23, «Cante y poesía», conferencia por Rafael León, con actuaciones musicales de Antonio Albaicín; y coloquio sobre el tema dirigido por Ángel Caffarena Such. Día 24, «Formación de los cantes primitivos», conferencia por Ricardo Molina, ilustrada por Antonio Mairena y coloquio sobre el tema dirigido por Manuel Agustín de Heredia. Día 25, «Ríos y afluentes del baile flamenco», conferencia por José Manuel Caballero Bonald, ilustrada por la Chunga; y coloquio sobre el tema dirigido por José Luque Navajas. Día 26, «Duende y misterio del flamenco», conferencia por Edgar Neville.

${ }^{7}$ Blog Papeles flamencos. http://www.papelesflamencos.com/2009/06/iii-semana-deestudios-flamencos-de.html. Último acceso: 18-02-2021. 
Se conserva una carta del 9 de agosto de 1964 en la que el cordobés le habla al jerezano de cómo Enrique Menor, flamencólogo, organizador de las semanas flamencas de Málaga y encargado de Relaciones Públicas de Coca Cola, empresa patrocinadora del evento, le ha propuesto que dirija un seminario sobre la «Soleá» («Málaga prepara la Segunda Semana»1964). Se expresa así:

Ayer recibí afectuosa carta de Enrique Menor aceptando mis condiciones pero proponiéndome que además de la conferencia asuma la dirección del Seminario flamenco que dedicará a la 'Soleá' la Semana del 20- al 26 de Sepbre. Esto es cosa nueva que me exige una estancia de 8 días en Málaga, preparar un programa exhaustivo a ser posible de temas y problemas a tocar y aceptar una responsabilidad nueva.

Le he contestado aceptando la conferencia y pidiéndole, aparte de las $10.000 \mathrm{pts,}$ 7.000 por encargarme del Seminario y que me paguen además alojamiento en $\mathrm{H}$. Miramar del 19 al 27.

Mis proyectos iniciales eran llegar a Málaga el 21 por la noche para volver a Córdoba el 23 por la mañana.

Deberé, además, buscar suplentes que me sustituyan en las 6 horas diarias de clase de $6^{\circ}$ curso y Preuniversitario, lo que me importará unas 3.000 pts.

Sin dudas, a la altura de 1964, después de muchos años alrededor del Concurso de Cante de Córdoba y con la salida reciente de Mundo y formas del cante flamenco, Molina tiene predicamento entre los organizadores de eventos. De ahí que sea invitado a dirigir el seminario sobre la soleá.

La Semana de Estudios Flamencos cuenta con el patrocinio de Coca Cola y esto sirve para que, por ejemplo, se convoquen concursos de carteles para anunciar el encuentro ( $A B C$, Madrid, 2 de mayo de 1964, p. 54) y becas de estudio sobre motivos flamencos. En la carta del 12 de octubre de 1963, es decir, antes de la celebración de la I Semana, Ricardo Molina introduce la beca de investigación como nuevo asunto en la correspondencia con Caballero Bonald. Molina le escribe:

No tenía noticia de la convocatoria de ayuda de investigación y como recibí tu carta ayer y no encontré quien me pasara a máquina el proyecto, lo tuve que enviar, con esta fecha, manuscrito por triplicado a la Casa de la Cultura. Le adjunté una carta al Presidente de la Comisión Organizadora suplicándole que dadas las circunstancias admitiera a concurso los manuscritos ( $¡ U n$ alarde caligráfico y cromático!)

El proyecto especifica los temas de mi investigación que formarían un libro titulado «Los Grandes Problemas del Cante Flamenco». Confío en que harás algo en favor mío, si tú mismo no concursas.

A pesar de todos sus esfuerzos, el cordobés no tiene suerte. Las premuras no le benefician: redacta y transcribe el proyecto en unas horas, lo envía el 12 o 13 de octubre de 1963 a solo diez días del comienzo de la Semana de Estudios, que arranca el lunes 21 de octubre. Así que en esta primera edición se 
hace con la beca Fernando Quiñones por fallo de un jurado compuesto por Juan Temboury, Caballero Bonald, Edgar Neville y Luis Caffarena Such, entre otros. De esa ayuda surge el libro de Quiñones De Cádiz y sus cantes (1964). Al año siguiente Molina lo vuelve a intentar en la convocatoria de la II Semana de Estudios Flamencos de Málaga que se celebra entre el 21 y el 26 de setiembre de 1964. Le escribe a Caballero Bonald el 9 de agosto de 1964 en los siguientes términos:

\begin{abstract}
Deseo que con toda franqueza me digas si estimas oportuno que concurse este año a la Beca de Investigación, pues ya sabes que el año pasado se consideró como mérito no haber hecho nada, ya que se me explicó que no se me había otorgado porque yo ya lo había dicho 'todo' cuanto tenía que decir en «Mundo y formas del cante flamenco» entonces en prensa. Si hay predisposición a favor de alguien dímelo para no molestarme en aspirar a la Beca.
\end{abstract}

Las cosas salen bien en esta segunda convocatoria y Ricardo Molina se hace con la beca en 1964. En una nota de prensa de La Vanguardia del 19 de setiembre de 1964, en la que se anuncia el programa de actos de la II Semana de Estudios Flamencos, se especifica el alcance de la Beca: «Por último se proclamará y entregará la beca de investigación, dotada con 50.000 pesetas, para premiar al mejor trabajo sobre el tema general "Cante, toque y baile flamencos”» («Homenaje a Pastora Imperio en la II Semana de Estudios Flamencos» 1964). Una vez desarrollado el proyecto presentado, Molina publica en la editorial Sagitario de Barcelona Misterios del arte flamenco. Ensayo de una interpretación antropológica (1967), un título en el que influye la opinión de Fernando Quiñones, asesor por entonces de dicha editorial, según apreciamos en dos cartas suyas dirigidas al cordobés del 19 y el 25 de octubre de 1967 (Rendón Infante 2011) y leemos en la nota que el gaditano antepuso al libro en su reedición de 1985 (Quiñones 1985).

Precisamente Ricardo Molina remata la carta a Caballero Bonald del 9 de agosto de 1964, previa a la celebración de la II Semana de Estudios Flamencos, en la que se haría con la beca, comentándole que lleva tiempo trabajando en un análisis sociológico y antropológico del flamenco. Esto no es más que el embrión del proyecto becado y del futuro libro Misterios del arte flamenco. Ensayo de una interpretación antropológica publicado en 1967. En la carta muestra su sorpresa por la indiferencia del cante flamenco ante las injusticias y quizás parezca posicionarse a favor de una actitud menos quejumbrosa. Este juicio entronca con el compromiso sociopolítico de algunos flamencólogos (Francisco Moreno Galván, Caballero Bonald, Félix Grande, José Manuel Flores), letristas (Manuel Gerena, Luis Marín, Andrés Ruiz, Paco Moyano) y cantaores (José Menese, Diego Clavel, Enrique Morente, Manuel Gerena, Amos Rodríguez Rey, Lola Hisado, Alfredo Arrebola) a partir de mediados de los años sesenta. Ricardo Molina se expresa así: 
Me interesan tus puntos de vista respecto al flamenco. Yo archivo y anoto desde hace más de tres años notas para una Sociología y Antropología del Arte Flamenco y me asusto de las conclusiones verdaderamente pesimistas a que conducen. El flamenco es en el fondo algo más que expresión de un pueblo subyugado: Es expresión de un pueblo capado. Solo así se explica su abulia, su indiferencia y su pasividad fatalista ante el reino de la injusticia económica, social y religiosa que es nuestra Andalucía. Aquí nunca hubo civismo ni espíritu de rebeldía. Los parias gitanos indostánicos se entendieron fácilmente con los parias andaluces del campo y la ciudad. Sobre estos asuntos me gustaría mucho hablar contigo y del cante como vehículo futuro de actitudes más gallardas, menos quejumbrosas y más esperanzadas...

Para terminar, he de decir que solo he hallado una carta de Caballero Bonald en el archivo de Ricardo Molina, la envía desde Madrid y la fecha el 17 de abril de 1965. En ella le agradece al cordobés su mediación para que asista al concurso de Arte Flamenco ${ }^{8}$. Dice lo siguiente:

Recibí hace unos días una amable carta del Alcalde de Córdoba, notificándome que mi nombre estaba entre los invitados de honor para asistir a las pruebas finales del IV Concurso Nacional de Arte Flamenco. Ya le contesté, aceptando la invitación y agradeciéndole la deferencia. Pero sé, naturalmente, que la iniciativa de todo esto ha partido de ti y no quiero dejar de testimoniarte mi mejor reconocimiento y amistad.

Nos veremos, pues, en Córdoba entre los días 19 y 21 de mayo. Tengo un especialísimo interés en asistir a esas pruebas del Concurso que con tan manifiesta primacía y eficacia vienes organizando.

La carta pone de manifiesto dos de las ideas matrices que han guiado este trabajo. Por un lado, evidencia la amistad invariable entre Ricardo Molina y José Manuel Caballero Bonald desde los albores de 1950 hasta el fallecimiento del primero en enero de 1968. Y, por otro, corrobora que, más allá de las relaciones que ambos mantienen como poetas, el verdadero y singular lazo de unión viene dado por sus respectivas devociones flamencas.

\section{FUENTES}

Acquaroni, José Luis. 1963. «En "Málaga cantaora”». $A B C$ (Madrid), 26 de octubre, pp. 33-34. Caballero Bonald, José Manuel. 1953. El cante andaluz. Madrid: Publicaciones Españolas. Caballero Bonald, José Manuel. 1956. Anteo. Palma de Mallorca: Ed. Papeles de Son Armadans.

${ }^{8}$ Para conocer el origen, el devenir y los entresijos del concurso de Córdoba, conviene acudir al libro de Agustín Gómez Pérez (2006). Ahí dedica un capítulo a esta cuarta edición, donde recoge el elenco de nombres invitados y los de aquellos que ratifican su asistencia por carta, como es el caso presente de Bonald. 
Caballero Bonald, José Manuel. 1957. El baile andaluz. Barcelona: Noguer.

Caballero Bonald, José Manuel. 1968. Archivo del cante flamenco. Barcelona: Ariola-Vergara.

Caballero Bonald, José Manuel. 1975. Luces y sombras del flamenco. Barcelona: Lumen.

Caballero Bonald, José Manuel. 2004. Tiempo de guerras perdidas. La novela de la memoria, I. Madrid: Alfaguara.

Caballero Bonald, José Manuel. 2008. «Ricardo Molina y el flamenco». En Ricardo Molina: conciencia de Cántico, ed. Antonio Rodríguez Jiménez, 99-108. Iluminaciones, 41. Sevilla: Renacimiento.

Cifra. 1964. «Clausura de la II Semana de Estudios Flamencos». $A B C$ (Madrid), 27 de setiembre, p. 93.

Díaz-Cañabate, Antonio. 1963. «Las "siguiriyas" de Manuel Torres». ABC (Edición de Andalucía), 26 de octubre, pp. 63-64.

«Homenaje a Pastora Imperio en la II Semana de Estudios Flamencos». 1964. La Vanguardia, 19 de setiembre, p. 7.

«Homenaje a Pastora Imperio». 1964. La Vanguardia, 27 de setiembre, p. 7.

Jiménez Martos, Luis. 1990. «Principio y fin de Cántico». Córdoba, 4 de mayo, p. 3.

«Málaga prepara la Segunda Semana de Estudios Flamencos. El poeta cordobés Ricardo Molina presidirá el Seminario de Estudios sobre la soleá» (1964). Córdoba, 26 de agosto, p. 5 .

Molina, Ricardo. 1951a. «Oda a Luis Cernuda». Platero 1, enero, pp. 2-3. Recogido en Cántico, 9-10, agosto-noviembre de 1955; Cántico. Hojas de Poesía. Córdoba, 19471957. Córdoba: Diputación de Córdoba, 1983, edición facsímil, pp. 384-385.

Molina, Ricardo. 1951b. «Tristán». Platero 6, junio, p. 9.

Molina, Ricardo. 1952. «Primeras lluvias». Platero 14, febrero, pp. 8-9.

Molina, Ricardo. 1965. Cante flamenco. Antología. Madrid: Taurus.

Molina, Ricardo. 1967. Misterios del arte flamenco. Ensayo de una interpretación antropológica. Barcelona: Sagitario.

Molina, Ricardo. 1977a. Cante y Cantaores cordobeses. Madrid: Ediciones Demófilo.

Molina, Ricardo. 1977b. Obra flamenca. Madrid: Ediciones Demófilo.

Molina, Ricardo. 1982. Homenaje. En Obra poética completa, II. Granada: Antonio Ubago.

Molina, Ricardo. 1992. Cartas de Ricardo Molina a Anselmo González Climent. Córdoba: Publicaciones del Ayuntamiento de Córdoba.

Molina, Ricardo. 2007. Homenaje. En Obra poética. Poesía póstuma, II. Madrid: Visor.

Molina, Ricardo y Antonio Mairena. 1963a. «El cante flamenco y las letras españolas contemporáneas». Cuadernos Hispanoamericanos 163-164: 108-123.

Molina, Ricardo y Antonio Mairena. 1963b. Mundo y formas del cante flamenco. Madrid: Revista de Occidente.

Tijeras, Eduardo. 1964. «A orillas del mediterráneo». ABC (Madrid), 11 de octubre, pp. 114 y 110.

\section{BiBLIOGRAFÍA CITADA}

Blas Vega, José y Manuel Ríos Ruiz. 1988. Diccionario enciclopédico ilustrado del flamenco. Madrid: Cinterco.

Carnero, Guillermo. 1976. El grupo «Cántico» de Córdoba. Un episodio clave de la historia de la poesía española de postguerra. Estudio y Antología. Madrid: Editora Nacional. 
Carnero, Guillermo. 2008. «Ricardo Molina, motor y conciencia de Cántico». En Ricardo Molina: conciencia de Cántico, ed. Antonio Rodríguez Jiménez, 21-35. Iluminaciones, 41. Sevilla: Renacimiento.

García Baena, Pablo. 2007. «La noche pasajera». En Silva varia, 142-146. Benalmádena: Eda.

Gómez Pérez, Agustín. 2006. Los concursos de Córdoba (1956-2006) (Análisis y comentarios). Córdoba: Ediciones de La Posada.

Gómez Pérez, Agustín. 2008. «Ricardo Molina y el flamenco». En Ricardo Molina: conciencia de Cántico, ed. Antonio Rodríguez Jiménez, 119-135. Iluminaciones, 41. Sevilla: Renacimiento,

González Climent, Anselmo. 1965. Bibliografía Flamenca. Madrid: Escelicer.

Linares, Abelardo. 1983. «Prólogo». En Cántico. Hojas de Poesía. Córdoba, 1947-1957. Córdoba: Diputación de Córdoba.

Neira, Julio. 2014. Memorial de disidencias (Vida y obra de José Manuel Caballero Bonald). Sevilla: Fundación José Manuel Lara.

Quiñones, Fernando. 1964. De Cádiz y sus cantes. Barcelona: Seix Barral.

Quiñones, Fernando. 1985. «Nota en 1985». En Misterios del arte flamenco. Ensayo de una interpretación antropológica, Ricardo Molina, 9-11. Barcelona: Editoriales Andaluzas Unidas.

Rendón Infante, Olga. 2011. «Cuatro cartas inéditas de Fernando Quiñones a Ricardo Molina». Cuadernos Hispanoamericanos 734: 71-92.

Rendón Infante, Olga. 2015. Los poetas del 27 y el grupo Cántico de Córdoba. Sevilla: Alegoría.

Torre, José María de la. 1995. Ricardo Molina, biografía de un poeta. Córdoba: Publicaciones Obra Social y Cultural de Cajasur.

Fecha de recepción: 27 de febrero de 2018.

Fecha de aceptación: 31 de mayo de 2018. 